\title{
THE ZEEMAN EFFECT REVISITED
}

\author{
J. AVRON ${ }^{1}$ and I. HERBST ${ }^{2}$ \\ Joseph Henry Laboratories of Physics, Princeton University, Princeton, New Jersey,08540, USA \\ B. $\operatorname{SIMON}^{3}$ \\ Belfer Graduate School of Science, Yeshiva University, New York, New York 10033, USA
}

Received 7 June 1977

\begin{abstract}
We announce three new rigorous results for the quantum mechanical hydrogen atom in constant magnetic field: (i) Borel summability of the small field perturbation series, (ii) detailed large field asymptotics, and (iii) non-degeneracy of the ground state $\Omega_{0}$ and a proof that it has $L_{z} \Omega_{0}=0$ for all values of the field.
\end{abstract}

The weak field Zeeman effect [1] in simple atoms was one of the earliest problems studied [2] in quantum mechanics. More recently, Ruderman [3] and then others [4] discussed the analogous problem in super-strong magnetic fields of the type encountered in neutron stars. It is perhaps surprising that any problems remain open for such a well studied theory but there are some unresolved theoretical questions of interest: (i) The Rayleigh-Schrödinger perturbation coefficients for the energy levels almost surely [5] diverge as $n$ ! Do they nevertheless determine the answer in some way? (ii) Is there a systematic large $B$ expansion for the ground state energy beyond the $c B$ and $\mathrm{d} \ln ^{2} B$ terms of ref. [4]? (iii) There are central potentials [6] where the ground state fails to be $m=0$ for $B$ in a suitable interval away from zero and is therefore degenerate for at least one value of $B$ by continuity [7]. Is the attractive Coulomb potential one of these or not?

We wish to describe here solutions of these three questions; full details of our results and methods will appear elsewhere [8]. Some of the results extend to more general atoms and we have studied the corrections due to finite nuclear mass [9] but we will state our results for the simple model:

$H(B)=(-\mathrm{i} \nabla-A)^{2}-r^{-1}$

${ }^{1}$ On leave from NRCN, Beer-Sheva, Israel. Research supported by NSF Grant MPS 74-22844 and MPS 75-22514.

${ }^{2}$ Research supported by NSF Grant MPS 74-22844.

${ }^{3}$ On leave from Departments of Mathematics and Physics, Princeton U. Research supported by NSF Grant MPS 75-11864.
$A=-\frac{1}{2}(r \times B) ; \quad B=(0,0, B)$

Theorem 1. Let $E_{n}(0)$ be any negative eigenvalue of the Hydrogen Hamiltonian $H(0)$. Then there is an eigenvalue [10] $E_{n}(B)$ of $H(B)$ for $B$ small which is the Borel sum $[11,12]$ of the Rayleigh-Schrödinger perturbation coefficients for $E_{n}$ [10].

Theorem 2 . The ground state [13] energy $E_{m}(B)$, of $H(B)$ restricted to the subspace with fixed azimuthal angular momentum $m$ is asymptotic for large $B$ and fixed $m$ to

$$
\begin{aligned}
& E_{m}(B)=B(|m|-m+1) \\
& \quad-\left[\frac{1}{2} \ln B-\ln (\ln B)+q_{|m|}+\mathrm{O}\left(\frac{\ln (\ln B)}{\ln B}\right)\right]^{2},
\end{aligned}
$$

where

$$
\begin{aligned}
& q_{m}=q_{0}-\frac{1}{2} \sum_{i=0}^{m-1} \frac{1}{i !(m-i)}, \quad m \geqslant 1 \\
& q_{0}=\frac{1}{2} \ln 2+\frac{1}{2} C+\varepsilon_{1}(1)+\int_{0}^{1} \frac{\mathrm{e}^{-x}-1}{x} \mathrm{~d} x \approx 0.05796,
\end{aligned}
$$

$C$ is Euler's constant and $\varepsilon_{1}(x)$ is the exponential integral.

Theorem 3. The ground state of $H(B)$ for any $B$ is non-degenerate and has $L_{z}=0$ [14].

The complete proofs of these results are too lengthy to give here but we can say something about the methods. To prove Theorem 1 [12] one needs to prove stability of the eigenvalues [15] for complex values of $B$ in some sec- 
tor. This turns out to be somewhat more subtle than the corresponding stability for the anharmonic oscillator [12]. By scaling (see eq. (3) below) and standard perturbation theoretic arguments, one shows that the domain of analyticity contains the cut plane intersected with a disc. The last element of the proof is the $n$ ! bound on the series expansion in a suitable region of the complex $B$ plane. Here we exploit a technique of Combes and Thomas [16] developed originally to prove the exponential falloff of bound state wave functions.

There is one very interesting aspect of our study of Theorem 1 we should mention: If $\psi_{0}$ is the ground state for $H(0)$, then $t^{-1} \ln \left(\psi_{0}, \mathrm{e}^{-t H(B)} \psi_{0}\right) \equiv \alpha_{t}(B)$ is a function with a formal perturbation series $\alpha_{t}(B)$ $=\Sigma a_{n}(t) B^{n}$ obeying $\left|a_{n}(t)\right| \leqslant A(t) B(t)^{n}(n / 2)$ ! despite the fact that the perturbation series $\Sigma a_{n}(\infty) B^{n}$ for $-E_{0}(B)=\lim _{t \rightarrow \infty} \alpha_{t}(B)$ undoubtedly has $\left|a_{n}(\infty)\right|$ $\sim n$ ! This example [17] is relevant to the Lipatov theory [18] of the asymptotics of the perturbation series for anharmonic oscillators and $\phi^{4}$ field theories where similar $t \rightarrow \infty$ and $n \rightarrow \infty$ limits are interchanged with abandon; we believe this interchange is correct in that case but it is clearly more subtle than previously believed.

To prove Theorem 2, one introduces a coupling constant $\lambda$ in front of the $r^{-1}$ term in (1) and notes that $E(B, \lambda)$, the ground state of $H(B, \lambda)$ obeys:

$E(B, 1)=B E\left(1, B^{-1 / 2}\right)$,

since $H(B, 1)$ and $B H\left(1, B^{-1 / 2}\right)$ are unitarily equivalent under the scaling $x \rightarrow B^{1 / 2} x$. Eq. (3) reduces the large $B$ behavior to a small coupling problem for $H(1,0)-\lambda r^{-1}$. Because the magnetic field in $H(1,0)$ discretizes the spectrum in two dimensions, this is essentially a small coupling problem in one dimension where systematic expansions have been recently developed [19].

Theorem 3 depends on certain monotonicity results obtained by developing a Wiener path integral for the Hamiltonian reduced to a fixed $m$ subspace, discretizing the corresponding "time" and using correlation inequalities [20] for the corresponding Ising-like system [21]. The Coulomb potential $V(r)=-r^{-1}$ is distinguished from the potentials of ref. [6] by the conditions $V^{\prime} \geqslant 0, V^{\prime \prime} \leqslant 0$. The critical input is a proof that under certain circumstances the ground state wave function of a quantum mechanical particle must collapse towards the origin as the potential becomes more attractive.

\section{References}

[1] The natural unit for the field is $\sim 10^{9}$ Gauss so that conventional laboratory fields are "small".

[2] W. Heisenberg, P. Jordan, Zeit. für Phys. 37 (1926) 263.

[3] R. Cohen, L. Lodenquai, M. Ruderman, Phys. Rev. Lett. 25 (1970) 467;

B.B. Kadomtsev, Sov. Phys. JETP 31 (1970) 945;

M. Ruderman, Phys. Rev. Lett. 27 (1971) 1306.

[4] R.O. Muller, R.P. Rau, L. Spruch, Phys. Rev. Lett. 26 (1971) 1136, Phys. Rev. A 11 (1975) 789, 1865, Astrop. J. 207 (1976) 671.

[5] The $n$ ! comes from the following: The leading term in Rayleigh-Schrödinger theory is $\alpha_{n}=\left(\Omega_{0}, V\left(S_{0} V\right)^{n-1} \Omega_{0}\right)$, where $S_{0}=\left(H_{0}-E_{0}\right)^{-1}\left[1-\left(\Omega_{0}, \cdot\right) \Omega_{0}\right] \cdot \Omega_{0} \sim \mathrm{e}^{-|x|}$ at infinity, $S_{0}$ has no falloff in $x$ and $V \sim|x|$ so $\alpha \sim \int|x|^{n} \mathrm{e}^{-x}$ $\sim n$ !

[6] R. Lavine and M. O'Carroll, to be published.

[7] The usual proof of the nodeless nature of the ground state depends on the fact that the Green's function $\equiv$ kernel of $(H-E)^{-1},\left(E<E_{0}\right)$ is strictly positive. This fails for Hamiltonians in magnetic fields where this Green's function is no longer even real.

[8] J. Avron, I. Herbst and B. Simon, Schrödinger operators in magnetic fields, in preparation.

[9] The reduction of the center of mass is actually subtle; for a free particle in a magnetic field, the momentum perpendicular to the field is not conserved but the gauge invariant quantity $C=p+e A$ (with $e$ the charge of the particle) related to the position of the center of rotation, is conserved and plays a role similar to the momentum. But $\left[C_{x}, C_{y}\right] \neq 0$. For a multiparticle system with pair interactions $C=\Sigma_{i} C_{i}$ is conserved. $C_{x}$ and $C_{y}$ will commute if and only if the total charge is zero. In this case a conventional kind of reduction is possible.

[10] Of course, the eigenvalues are highly degenerate. Our results hold for the states of maximal and minimal $m$ which are non-degenerate on the fixed $m$ subspace.

[11] That is, if $\Sigma a_{n} B^{n}$ is the formal perturbation series, then $g(x) \equiv \Sigma a_{n} x^{n} / n$ ! converges for $x$ small, has an analy tic continuation to a neighborhood of the positive reals and for $B$ small $E(B)=\int_{0}^{\infty} g(x B) \mathrm{e}^{-x} \mathrm{~d} x$.

[12] Borel summability methods for Rayleigh-Schrödinger series go back to S. Graffi, V. Grecchi and B. Simon, Phys. Lett. 32B (1970) 631, who prove this summability for the anharmonic oscillator. It has since been extensively developed in a variety of situations, including certain field theories; see e.g. I.P. Eckmann, I. Magnen and R. Sénéor, Commun. Math. Phys. 39 (1975) 251.

[13] All states but these states are of order $B(|m|-m+1)$ $+\mathrm{O}(1)$ as $B \rightarrow \infty$.

[14] This is true in any gauge for which $L_{z}$ is a constant of the motion.

[15] This is a technical condition which says that the eigenvalue remains isolated and its multiplicity constant for small complex $B$. An example of a non-stable perturbation, even for real coupling, is the Stark problem; see e.g. J. Avron and I. Herbst, Commun. Math. Phys. 52 (1977) 239. 
[16] J.M. Combes, L. Thomas, Commun. Math. Phys. 34 (1973) 250.

[17] A more artificial but even stronger example is to take $a_{n}(t)=(n / 2) !+\int \delta y^{n} \mathrm{e}^{-y} \mathrm{~d} y$, where $a_{n}(t) \sim(n / 2) !$ as $n \rightarrow \infty$ for any fixed $t<\infty$ but $a_{n}(\infty) \sim n !$ as $n \rightarrow \infty$.

[18] L.N. Lipatov, Pisma JETP 24 (1976) 179;

E. Brezin, J.C. LeGuillou and J. Zinn-Justin, Phys. Rev., in press.

[19] B. Simon, Ann. Phys. 97 (1976) 279;

R. Blankenbecler, M.L. Goldberger and B. Simon, Ann.
Phys., to appear. Their method must be extended to treat Coulomb tails.

[20] There is an extensive literature on correlation inequalities beginning with R. Griffiths, J. Math. Phys. 8 (1967) 478. We actually must prove a new kind of FKG inequality for a very special class of multicomponent models.

[21] The idea of using correlation inequalities for discretized path integrals is due to F. Guerra, L. Rosen and B. Simon, Ann. Math 101 (1975) 111, and has been extensively developed. 